\title{
ON QUASIHEREDITARY RINGS
}

\author{
W. D. BURGESS AND K. R. FULLER \\ (Communicated by Donald S. Passman)
}

\begin{abstract}
The notion of a quashereditary semiprimary ring was introduced by Cline, Parshall and Scott and was studied extensively by Dlab and Ringel. It is known that if $R$ is semiprimary of global dimension $\leq 2$ then it is quasihereditary and that there is a serial ring of global dimension 4 which is not. This paper establishes three principal results: If $R$ is quasihereditary then the Cartan determinant conjecture is true for $R$ (i.e., $\operatorname{det} C(R)=1$ ); a serial ring is quasihereditary iff it has a heredity ideal; in particular, every serial ring of global dimension 3 is quasihereditary; and there is a ring (a 0-relations algebra in fact) of global dimension 3 which is not quasihereditary.
\end{abstract}

\section{INTRODUCTION}

Throughout all rings, which are denoted by $R$, are assumed to be unitary and semiprimary (i.e., the radical $N$ is nilpotent and $R / N$ is semisimple artinian). Following [DR1] we say that an ideal $I$ of $R$ is heredity if $I^{2}=I,{ }_{R} I$ is projective and $I N I=0$. Further, $R$ is quasihereditary if there exists a chain of ideals $0=I_{0} \subset I_{1} \subset \cdots \subset I_{m}=R$ such that $I_{t} / I_{t-1}$ is a heredity ideal $R / I_{t-1}, t=1, \ldots, m$. (Such a chain is called a heredity chain.) Of course, if $m=1, R$ is semisimple. These rings were introduced by Cline, Parshall and Scott ([CPS]) in the context of representation theory. Their importance lies also in the fact every semiprimary ring is the endomorphism ring of a projective module over a quasihereditary ring, as was shown by Dlab and Ringel in [DR3]. They form a natural generalization of hereditary rings but as shown in [DR1] they can be of arbitrary finite global dimension.

All semiprimary rings of global dimension $\leq 2$ are quasihereditary ([DR2]) but [DR2] also contains an example of an 11 dimensional serial algebra over a field which is of global dimension 4 but is not quasihereditary. We show below (2.3) that a serial ring is quasihereditary iff it contains a heredity ideal and, as a consequence (2.7), that if $R$ is serial of global dimension 3 then it is quasihereditary. The example in section 3 shows that even a 0-relations algebra over a field of global dimension 3 need not be quasihereditary.

Received by the editors July 21, 1988.

1980 Mathematics Subject Classification (1985 Revision). Primary 16A35, 16A32.

Key words and phrases. Quasihereditary rings, Cartan determinant, serial ring.

The work of the first author was supported by Grant A7539 of the NSERC. 
Let $R$ be a basic left artinian ring with a basic set of primitive idempotents $e_{1}, \ldots e_{n}$. Then the (left) Cartan matrix $C(R)$ of $R$ is the $n \times n$ integral matrix whose $(\mathrm{i}, \mathrm{j})$-entry is the number of copies of the simple $S_{i}=R e_{i} / N e_{i}$ which occur in a composition series for $R e_{i}$. It has long been known that $\mathrm{gl}$. dim. $R<$ $\infty$ implies $\operatorname{det} C(R)= \pm 1$ and it is conjectured that finite global dimension implies $\operatorname{det} C(R)=1$. This is called the Cartan determinant conjecture (see [BFVZ] and [FZ] for recent information about it). It is shown below that the conjecture is true for quasihereditary rings.

We shall keep the above notation throughout: $e_{1}, \ldots, e_{n}$ is a basic set of primitive idempotents and $S_{1}, \ldots, S_{n}$ the corresponding simples.

\section{Preliminary Results and the Cartan Determinant}

We first show that if $R$ has a heredity ideal then it has one of the form $R e_{i} R$.

Lemma 1.1. Suppose that $I$ is a heredity ideal of $R$. Then $I$ can be written $I=R e R$ where (with suitable renumbering) $e=e_{1}+\cdots+e_{k}$. Then for each $j=1, \ldots, n, \operatorname{Re}_{1} \operatorname{Re}_{j}$ is either 0 or a direct sum of copies of $\operatorname{Re}_{1}$. In particular, $\operatorname{Re}_{1} R$ is heredity.

Proof. Since $I$ is idempotent it has the form $R e R$ for some $e=e^{2}$ (see e.g. [DR1]), but equivalent idempotents generate the same ideal, so $e$ may be taken to have the form $e_{1}+\cdots+e_{k}$, for some $k$. For any $j, R e R e_{j}$ is a direct summand of $I$, so it is projective and is generated by $R e$. Hence $\operatorname{Re} \boldsymbol{R e}_{j}=Q_{1} \oplus \cdots \oplus Q_{k}$, where each $Q_{i}$ is isomorphic to a direct sum of copies of $R e_{i}$. Since $e_{1}=e_{1} e, R e_{1} R e_{j}=R e_{1} R e R e_{j}$ and then $R e_{1} R e_{j}=\sum_{i=1}^{k} \oplus \operatorname{Re}_{1} Q_{i}$. Now $R e_{1} R e_{j}=0$ if $j=2, \ldots, k$ since $I N I=0$, giving that $R e_{1} R e_{j}=$ $\operatorname{Re}_{1} Q_{1}=Q_{1}$. Since $R e_{1} R e_{1}=R e_{1}, R e_{1} R$ is projective. Hence it is heredity. $\diamond$

Corollary 1.2. If $R$ is quasihereditary, the primitive idempotents can be numbered so that $0 \subset \operatorname{Re}_{1} R \subset R\left(e_{1}+e_{2}\right) R \subset \cdots \subset R\left(e_{1}+\cdots+e_{n}\right) R=R$ is a heredity chain.

Proof. Given a heredity chain $0=I_{0} \subset I_{1} \subset \cdots \subset I_{t}=R$, we have seen that we can write $I_{1}=R\left(e_{1}+\cdots e_{k}\right) R$ (with a suitable renumbering) and, by the lemma, $R e_{1} R$ is heredity. Now $0 \subset I_{1} / R e_{1} R \subset I_{2} / R e_{1} R \subset \cdots \subset R / R e_{1} R$ is a heredity chain and $e_{2}+R e_{1} R, \ldots, e_{n}+R e_{1} R$ is a complete orthogonal basic set of primitive idempotents for $R / R e_{1} R$. The process can be continued. $\diamond$

Note that if $R$ is quasihereditary then $\mathrm{gl} \cdot \operatorname{dim} . R \leq 2(n-1)$ and the Loewy length is $\leq 2^{n}-1$ by [DR 1 , Statement 9]. Of course this particular heredity chain may be longer than necessary but it does give bounds as functions of the number of basic primitive idempotents.

Proposition 1.3. Let $R$ be a left artinian ring. Let $C(R)$ be its Cartan matrix. If $R$ is quasihereditary then $\operatorname{det} C(R)=1$. 
Proof. Without loss of generality, we may take $R$ to be basic. Indeed the notion "quasihereditary" is a Morita invariant since the lattice isomorphism between the lattices of two Morita equivalent rings preserves multiplication of ideals. Hence a heredity chain is taken to a heredity chain. The Cartan matrix is also a Morita invariant.

Using the lemma, let $I=R e_{1} R$ be heredity. Since $e_{1} N e_{1}=0, c_{11}=1$ in $C(R)$. For $j>1, I e_{j} \cong\left(R e_{1}\right)^{m_{j}}$, again by the lemma. Hence elementary column operations transform $C(R)$ into the form

$$
\left[\begin{array}{cccc}
1 & 0 & \cdots & 0 \\
c_{21} & & & \\
\cdot & & C(R / I) & \\
\cdot & & &
\end{array}\right] .
$$

The result follows by induction on $n$ since when $n=1, R$ is simple. $\diamond$

Another result which may turn out to be true for all rings of finite global dimension is true for quasihereditary rings. It was established for global dimension $\leq 2$ in [GGZ].

Proposition 1.4. Let $R$ be a quasihereditary semiprimary ring. Then for an simple module $S, \operatorname{Ext}^{1}(S, S)=0$.

Proof. With the usual notation, we may assume that $I=R e_{1} R$ is heredity (by (1.1)). Then $e_{1} N e_{1}=0$, giving $\operatorname{Ext}^{1}\left(S_{1}, S_{1}\right)=0$. Now $\operatorname{Rad} R / I=(N+I) / I$ and if we work by induction on $n$ (again the result is clear when $n=1$ ), we get that

$$
\left(e_{i}+I\right) \frac{N+I}{I}\left(e_{i}+I\right) \subseteq \frac{N^{2}+I}{I}
$$

for $i>1$. That is, $e_{i} N e_{i} \subseteq N^{2}+I$ for $i>1$; then $e_{i} N e_{i} \subseteq e_{i} N^{2} e_{i}+e_{i} R e_{1} R e_{i} \subseteq$ $e_{i} N^{2} e_{i}$. Hence, again, $\operatorname{Ext}^{1}\left(S_{i}, S_{i}\right)=0$. $\diamond$

Before going on to the serial case, let us note that l-hereditary rings (and, in particular, finite dimensional incidence algebras) are quasihereditary. These are rings such that for any $i \neq j$ any $0 \neq h: R e_{i} \rightarrow R e_{j}$ is monic (see [M-V]). First there is a lemma which will be used again.

Lemma 1.5. Let $R e_{i}$ be simple. Then $R e_{i} R=I$ is hereditary. Further, if $e_{i} R$ is simple, then $\operatorname{Re}_{i} R$ is heredity.

Proof. Since $I \subseteq \operatorname{Soc}\left({ }_{R} R\right), N I=0$. Further $I$ is the $S_{i}$-homogeneous component of $\operatorname{Soc}\left({ }_{R} R\right)$. It is projective. The second part follows from [DR1, Statement 7] which shows that the notion of heredity ideal is left-right symmetric. $\diamond$

Proposition 1.6. If $R$ is a semiprimary ring which is l-hereditary then $R$ is quasihereditary. 
Proof. One sees readily that there are simple projectives. Suppose $R e_{1}$ is one. Then $I=R e_{1} R$ is heredity. Put $f=1-e_{1}$. The claim is that $R / I \cong f R f$. Indeed $r \rightarrow f r f$ induces a homomorphism since $f r s f=f r f s f+f r e_{1} s f$. The second term is zero since $N I=0$. The kernel is $I$ since if $f r f=0$ then $r=f r f+e_{1} r f+f r e_{1}+e_{1} r e_{1} \in I$. Now $f R f$ will also be $l$-hereditary so the process can continue. $\diamond$

In fact heredity ideals always give rise to primitive idempotents which behave like those of $l$-hereditary rings.

Lemma 1.7. Let $R$ be a semiprimary ring and $I=R e R$ a heredity ideal with $e=e_{1}+\cdots+e_{k}$. Then for each $i=1, \ldots, k$, any homomorphism $0 \neq h: \operatorname{Re}_{i} \rightarrow$ $R$ is monic.

Proof. Since $I$ is the trace of $R e, \operatorname{Hom}_{R}\left(\operatorname{Re}_{i} R\right)=\operatorname{Hom}_{R}\left(R e_{i}, I\right)$. Further the projective $I \cong\left(R e_{1}\right)^{m_{i}} \oplus \cdots \oplus\left(R e_{k}\right)^{m_{k}}:=P$, via, say, $q$. Now $e N e \subseteq I N I=0$, so if $\pi: P \rightarrow R e_{j}$ is a projection, $1 \leq j \leq k$, such that $\pi q h\left(e_{i}\right) \neq 0$ it follows that $\pi q h\left(e_{i}\right)=e_{i} \pi p h\left(e_{i}\right) e_{j} \notin N e_{j}$. But then $\pi q h: R e_{i} \rightarrow R e_{j}$ is an isomorphism and $i=j$. Hence $h$ is monic. $\diamond$

The converse is false however, even for diagram algebras ([F2]), as the following example shows.

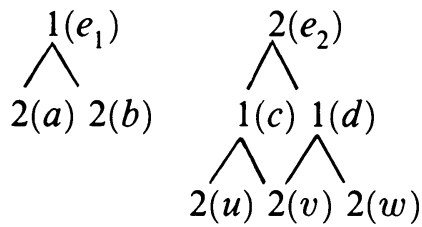

Let $R$ be the algebra over a field $K$ given by the accompanying diagram, where the numbers represent the simples in composition series for the indecomposable projectives and the letters are the elements of a multiplicative basis (see [F2] for more on this kind of presentation). The parts of the multiplication table which are not obvious from the diagrams are: $a c=v, b c=u, a d=w$ and $b d=v$. Then it is readily seen that $\operatorname{Hom}\left(R e_{1}, R e_{2}\right)=K c+K d$ and that any $0 \neq h: R e_{1} \rightarrow R e_{i}$ is monic for $i=1,2$. Equally clear is that $R e_{1} R$ is not projective.

The converse does hold for 0-relations algebras (see e.g. [GHZ] for the definition); i.e., if $0 \neq h: R e_{i} \rightarrow R e_{j}$ is monic for all $j$, then $R e_{i} R$ is heredity.

\section{QUASIHEREDITARY SERIAL RINGS}

In this section the notions and notations of [F1] are used. Two cases need separate study, when $R$ has a simple projective and when it does not.

Lemma 2.1. Let $R$ be an indecomposable serial ring. If $R$ has no simple projectives and $e_{i}$ is a primitive idempotent then $R / R_{i} R$ is indecomposable with a simple projective $\operatorname{Re}_{i+1}+\operatorname{Re}_{i} R$.

Proof. We arrange the primitive idempotents to give a Kupisch series ([F1]) and since there are no simple projectives we may assume $i \neq n$ (for convenience 
of notation). Now $\operatorname{Re}_{i} R e_{j} \subseteq N^{2} e_{j}$ for $j=i+2, \ldots, n, 1, \ldots, i-1$. If indicates "modulo $R e_{i} R$ ", it follows that $\bar{R} \bar{e}_{i+1}, \ldots, \bar{R} \bar{e}_{n}, \bar{R} \bar{e}_{1}, \ldots, \bar{R} \bar{e}_{i-1}$ is a Kupisch series for $\bar{R}$ and there is no non-zero map $\bar{R}_{i-1} \rightarrow \bar{R} \bar{e}_{i+1}$. Hence $\bar{R} \bar{e}_{i+1}$ is simple. $\diamond$

Lemma 2.2. Let $R$ be an indecomposable serial ring with a simple projective. Then $R$ is quasihereditary.

Proof. Let $R e_{1}, \ldots, R e_{n}$ be a Kupisch series where $R e_{1}$ is simple. Then by (1.5), $I=R e_{1} R$ is heredity. Further, $\bar{R} \bar{e}_{2}, \ldots, \bar{R} \bar{e}_{n}$ is a Kupisch series for $\bar{R}$ and it is clear that $\bar{R} \bar{e}_{2}$ is simple (the notation of the previous lemma is used). The process continues. $\diamond$

Proposition 2.3. An indecomposable serial ring $R$ is quasihereditary iff it has a heredity ideal.

Proof. One direction is obvious. For the other, if $R$ has a simple projective then (2.2) gives the result. If not, $R$ has a heredity ideal which may be taken to be of the form $R e_{i} R$ (1.1). Now (2.1) says that $R / R e_{i} R$ has a simple projective. $\diamond$

This result may be translated into terms of the composition lengths of the indecomposable projectives as follows. (Here $[m]$ is the class of $m$ modulo $n$ from among $1, \ldots, n$.)

Proposition 2.4. Let $R$ be an indecomposable serial ring. Then $R$ is quasihereditary iff a Kupisch series for $R$ may be written in such a way that there is a portion $\boldsymbol{R e}_{i}, \ldots, \boldsymbol{R e}_{i+k-1}$ with composition lengths $c_{i}<\cdots<c_{i+k-1}$ and $c_{[i+k]} \leq k$.

Proof. If there is such a portion, then $R e_{i} R e_{i+p} \cong R e_{i}$ for $p=1, \ldots, k-1$ while $\operatorname{Re}_{i} \operatorname{Re}_{j}=0$ if $j \neq i, i+1, \ldots, i+k-1$. Hence $\operatorname{Re}_{i} R$ is projective. Furthermore, $e_{i} N e_{i}=0$, since $N e_{i}$ is an epimorph of $R e_{[i-1]}$.

In the other direction if $R e_{i} R$ is heredity, then $h: R e_{i} \rightarrow R e_{j}$ is either 0 or is monic. This readily translates into the condition. $\diamond$

We have already seen how a simple projective gives a heredity ideal. The next lemma shows that in the serial case a simple of projective dimension 2 also plays a crucial rôle.

Lemma 2.5. If $R$ is an indecomposable serial ring which has a simple of projective dimension 2 then it is quasihereditary.

Proof. It suffices by (2.3) to find a heredity ideal. Suppose p. $\operatorname{dim} S_{i}=2$. Let $R e_{1}, \ldots R e_{n}$ be Kupisch series chosen for convenience so that $i=1$. Set $k=$ $c_{i}$, the composition length of $R e_{i}$. We have an exact sequence $0 \rightarrow N^{k-1} e_{i-1} \rightarrow$ $R e_{i-1} \rightarrow R e_{i} \rightarrow S_{i} \rightarrow 0$, and, by hypothesis, $N^{k-1} e_{i-1}$ is projective. By [F1, Lemma 2.1], $N^{k-1} e_{i-1} \cong R e_{[i-k]}$. It follows that $c_{i-1}=c_{[i-k]}+k-1$. but there are always the inequalities $c_{i-1} \leq c_{[i-2]}+1 \leq c_{[i-3]}+2 \leq \cdots \leq c_{[i-k]}+k-1(=$ $\left.c_{i-1}\right)$. Hence, $c_{i-1}=c_{[i-2]}+1=\cdots=c_{[i-k]}+k-1$, and, in particular, $c_{i-1}>c_{[i-2]}>\cdots>c_{[i-k]}>\cdots>c_{[i-k]}$. Now since $c_{i}=k,(2.4)$ gives the result. $\diamond$ 
In order to use this in the case of global dimension 3 we also need the following:

Lemma 2.6. If $R$ is any left artinian ring of left global dimension $\leq 3$ then it has a simple projective dimension 0 or 2 .

Proof. We recall the following ([M, p. 204, Ex . 3]). If $0 \rightarrow A \rightarrow B \rightarrow C \rightarrow 0$ is exact then

(i) p. $\operatorname{dim} \cdot A>$ p. $\operatorname{dim} . B \Rightarrow$ p. $\operatorname{dim} \cdot C=$ p. $\operatorname{dim} \cdot A+1$, and

(ii) p. $\operatorname{dim} . A<\mathrm{p} . \operatorname{dim} . B \Rightarrow$ p. $\operatorname{dim} . C=$ p. $\operatorname{dim} . B$.

Now suppose that p. $\operatorname{dim} . S_{i}=3$ and let $0=K_{0} \subset K_{1} \subset \cdots \subset K_{s}=N e_{i} \subset$ $R e_{i}$ be the composition series for $R e_{i}$ and set $T_{j}=K_{j} / K_{j-1}$ and $L_{j}=R e_{i} / K_{j}$. Suppose further that no simple is of projective dimension 0 or 2 . From the exact sequence $0 \rightarrow T_{1} \rightarrow R e_{i} \rightarrow L_{1} \rightarrow 0$ and i) we get, using the fact that gl. $\operatorname{dim} . R=3$ and the hypothesis, p. $\operatorname{dim} . T_{1}=1$ and p. $\operatorname{dim} . L_{1}=2$. Now from the exact sequence $0 \rightarrow T_{2} \rightarrow L_{1} \rightarrow L_{2} \rightarrow 0$ we must have p. $\operatorname{dim} . T_{2}=1$ and by ii) p. $\operatorname{dim} \cdot L_{2}=2$. Continuing in this fashion we arrive at the exact sequence $0 \rightarrow T_{s} \rightarrow L_{s-1} \rightarrow L_{s} \rightarrow 0\left(L_{s}=s_{i}\right)$ and, as above, p.dim. $L_{s}=2$. But this is a contradiction.

Proposition 2.7. Let $R$ be a serial ring of global dimension $\leq 3$. Then $R$ is quasihereditary.

Proof. Lemmas 2.2, 2.5 and 2.6 give the result.

It is quite easy to reduce the case of a left serial ring to that of a serial ring. As pointed out in [BFVZ, p . 160], the (left) quiver of an indecomposable serial ring is either a rooted tree or like a rooted tree but with the root replaced by an oriented cycle. The tips of the branches represent simple projectives and, hence, give heredity ideals. More precisely, if there are no arrows into $i$ in the quiver, say with $i \rightarrow j \ldots$, then $R e_{i} R$ is heredity and dividing by it has the effect of pruning the twig $i \rightarrow$ from the quiver. This procedure may be continued until the quiver is that of a serial ring and (2.3) or (2.4) may be used.

\section{GLOBAL DIMENSION 3 DOES NOT IMPLY QUASIHEREDITARY}

The sole purpose of this section is to present an example of a 0-relations algebra $R$ of global dimension 3 which is not quasihereditary*. The language of [F2] is used and the algebra is presented as a diagram algebra as in the example of Section 1. Here $n=5$, the Loewy length is 6 and the algebra is of dimension 33 over any field $K$. In this case there is no ambiguity about the multiplication table for the multiplicative basis so the symbols for the basis

*The authors have been informed that $\mathrm{K}$. Yamagata has also found on example of an algebra of global dimension 3 which is not quasihereditary. 
elements have been omitted.

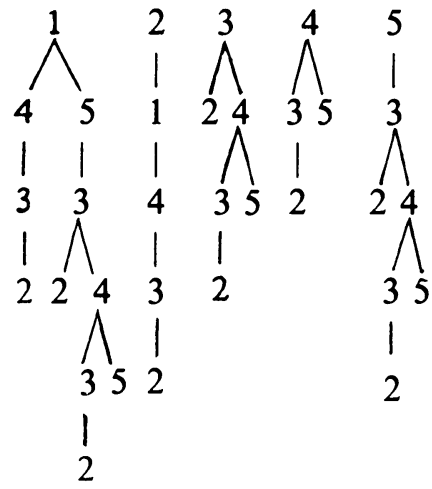

Projective resolutions for the simples can be read off from the diagrams (see [F2, Theorem 2.5]).

$$
\begin{array}{r}
0 \rightarrow R e_{3} \rightarrow R e_{5} \rightarrow R e_{4} \oplus R e_{5} \rightarrow R e_{1} \rightarrow S_{1} \rightarrow 0 \\
0 \rightarrow R e_{5} \rightarrow R e_{1} \rightarrow R e_{2} \rightarrow S_{2} \rightarrow 0 \\
0 \rightarrow R e_{5} \rightarrow R e_{1} \rightarrow R e_{2} \oplus R e_{4} \rightarrow R e_{3} \rightarrow S_{3} \rightarrow 0 \\
0 \rightarrow R e_{3} \oplus R e_{4} \rightarrow R e_{3} \oplus R e_{5} \rightarrow R e_{4} \rightarrow S_{4} \rightarrow 0 \\
0 \rightarrow R e_{3} \rightarrow R e_{5} \rightarrow S_{5} \rightarrow 0
\end{array}
$$

Moreover, $R$ is a 0-relations algebra by [F2,Theorems 3.3 and 4.2] and it is apparent that each $R e_{i} R$ contains a proper epimorph of $R e_{i}$ as a direct summand, so $R$ is not quasihereditary.

Added in proof: the authors have recently found an example of the type in $\S 3$ with $n=2$ and $N^{4}=0$.

\section{REFERENCES}

[BFVZ] W. D. Burgess, K. R. Fuller, E. R. Voss and B. Zimmerman-Huisgen, The Cartan matrix as an indicator of finite global dimension for artinian rings, Proc. Amer. Math. Soc. 95 (1985), 157-165.

[CPS] E. Cline, B. Parshall and L. Scott, Finite dimensional algebras and highest weight categories, Crelles J. Math., to appear.

[DR1] V. Dlab and C. Ringel, Heredity ideals, typescript 1987, 11 pages.

[DR2] _ Q Quasi-hereditary algebras, typescript 1987, 7 pages.

[DR3] _ Every semiprimary ring is the endomorphism ring of a projective module over a quasihereditary ring, typescript 1987,7 pages.

[F1] K. R. Fuller, Generalized uniserial rings and their Kupisch series, Math. Zeit. 106 (1968), 248-260.

[F2] _ Algebras from diagrams, J. Pure Appl. Algebra 48 (1987), 23-37.

[FZ] K. R. Fuller and B. Zimmermann-Huisgen, On the generalized Nakayama conjecture and the Cartan determinant problem, Trans. Amer. Math. Soc. 294 (1986), 679-691.

[GGZ,] E. L. Green, W. Gustafson and D. Zacharia, On artinian rings of global dimension two, J. Algebra 92 (1985), 375-379. 
[GHZ] E. L. Green, D. Happel and D. Zacharia, Projective resolutions over artin algebras with zero relations, Ill. J. Math. 29 (1985), 180-190.

[M] S. Mac Lane, Homology, Springer-Verlag, New York, 1967.

[M-V] R. Martinez-Villa, Algebras stably equivalent to l-hereditary algebras, Lecture Notes in Math. no. 832, Springer-Verlag, New York, 1980, 396-431.

Department of Mathematics, University of Ottawa, Ottawa, Ontario, Canada K1N 6N5

Department of Mathematics, University of Iowa, Iowa City, Iowa 52242 